\title{
Design and evaluation of a detection system for uniform dressing/undressing using intra-body communication technology
}

\author{
M. Fujikawa ${ }^{1,5}$, M. Nishigaki ${ }^{2}$, M. Yoshizawa ${ }^{3}$, K. Furusawa $^{4}$ \\ \& S. Tsujii ${ }^{5}$ \\ ${ }^{I}$ Sohgo Security Services Co., Ltd. (ALSOK), Japan \\ ${ }^{2}$ Shizuoka University, Japan \\ ${ }^{3}$ Tokyo Metropolitan College of Industrial Technology, Japan \\ ${ }^{4}$ Mitsuya Laboratories Inc., Japan \\ ${ }^{5} R \& D$ Initiative, Chuo University, Japan
}

\begin{abstract}
The authors developed a new uniform dressing/undressing detection system. This system utilizes intra-body communication technology in the form of a sensor to detect whether the uniform is on or not. Problems associated with conventional uniform dressing/undressing detection systems using ultrasonic sensors are eliminated owing to the use of intra-body communication technology. High practicality, reliability, and the safety of the proposed system were demonstrated by installing and experimenting the system in an actual uniform.
\end{abstract}

Keywords: social engineering, intra-body communication, abuse of uniforms.

\section{Introduction}

Crimes of swindling money and valuables from victims by posing as officials wearing illegally obtained occupational uniforms or imitations of genuine uniforms are frequent everywhere in the world and at all times [1,2]. This is a crime effectively utilizing the assumption that those in official uniforms are genuine officials, and in the study of security, it is called "social engineering" $[3]$. 


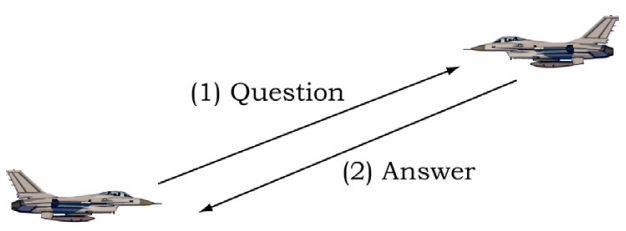

(3) Friend !

Figure 1: Image diagram of the IFF system.

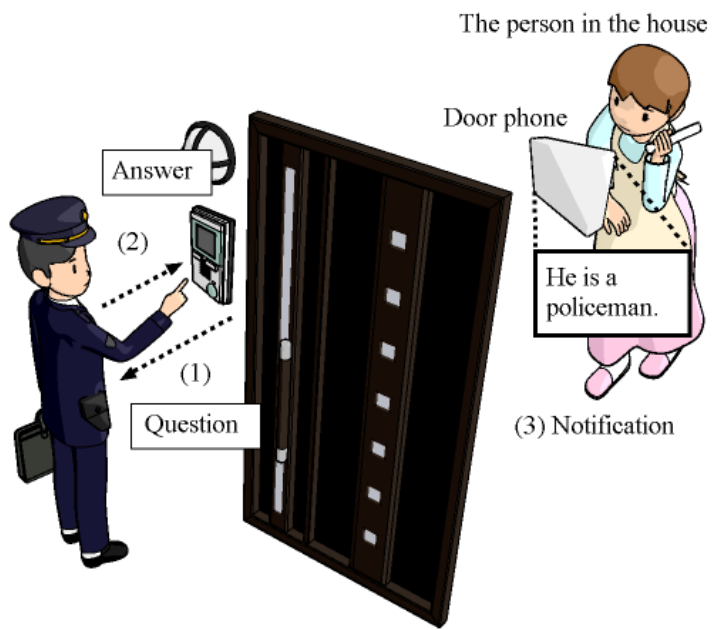

Figure 2: Incorporation into the door phone system.

In the military field of application, the IFF system of quickly identifying whether specific approaching aircraft, aiming aircraft or vessels are friend or foe is an established technology [4]. In this system, an interrogating signal is transmitted to the approaching aircraft requesting a response signal. If the received response signal is correct, the system determines that the approaching aircraft is a friend, and if the response signal is not correct or no signal is received, the system determines that the approaching aircraft is a hostile. For example as shown in Figure 2, when such a system is incorporated into a police officer's uniform and a door phone system is used, the person in the house may be able to identify whether the visitor is a genuine police officer or not.

Prerequisites: a door phone system should be connected to a secure network with constant access to information about the uniform of the police officer. The system should be able to determine the authenticity of the person ringing the bell and wearing a police officer's uniform and to notify the person in the house of the authentication. No person other than a police officer will have the uniform.

With such a system, it is possible to cope with social engineering when someone uses a uniform imitating an occupational uniform; however, because of social engineering, someone using a genuine uniform cannot be recognized as 
impersonating an official. Considering that genuine uniforms are sold via Internet auction sites [5], social engineering may occur when someone uses the genuine occupational uniform. Accordingly the authors consider it necessary to develop a system with the following functions.

1. Uniform " $\mathrm{A}$ " can recognize whether a person wearing Uniform " $\mathrm{A}$ " is genuinely Employee "A".

2. Uniform " $\mathrm{A}$ " activates a function demonstrating that Employee " $\mathrm{A}$ " is genuinely Employee " $\mathrm{A}$ " as far as he/she is wearing Uniform " $\mathrm{A}$ ".

3. Anyone can easily recognize whether an employee wearing a uniform is the genuine employee.

The authors started the study on the uniform dressing/undressing detection system that will become the basis of such a system and developed a system using an ultrasonic sensor [6]. However, there were some flaws in the developed system, for example, whether the uniform is worn cannot be recognized. So in this paper, a new uniform dressing/undressing detection system is proposed. Intra-body communication technology is employed in this new system as a sensor to detect whether the uniform is put on or not. Experiments conducted to verify the practicality, reliability, and safety of the system and discussions on the results of experiments are included in the latter half of this paper.

\section{Proposed system}

\subsection{Intra-body communication technology}

Intra-body communication technology was developed as a communication technology using the human body as communication path based on its ability to hold a static charge and to conduct a slight electric current. Among several methods so far proposed [7-9], the static coupling method is used in this study (see Appendix). The reasons why the static coupling method is used are as follows.

1. Electrodes are not necessarily in contact with the human body.

2. Communication can be established even when the electrodes are covered with a thin layer of insulation material.

3. Experiments are possible with the simple configuration of equipment, and inexpensive installation of the devices is possible.

\subsection{Detection mechanism of dressing/undressing}

In this section, the mechanism of detecting dressing/undressing is explained. First, the explanation of the method of installation of the dressing/undressing mechanism (see Figure 3) and functions of the detector are provided.

A detector, transmitting electrode, and receiving electrode are connected to a shielded wire, and each electrode is installed on each side of the shoulders of the upper garment. This is because it was shown after an investigation that mostly both shoulders are in contact with the upper garment of the uniform (half sleeves or long sleeves) when worn. The detector sends a signal to the transmitting 
electrode applying a slight alternating current. The detector amplifies the received signal with the receiving electrode and compares it to the transmitted signal and determines whether both signals match.

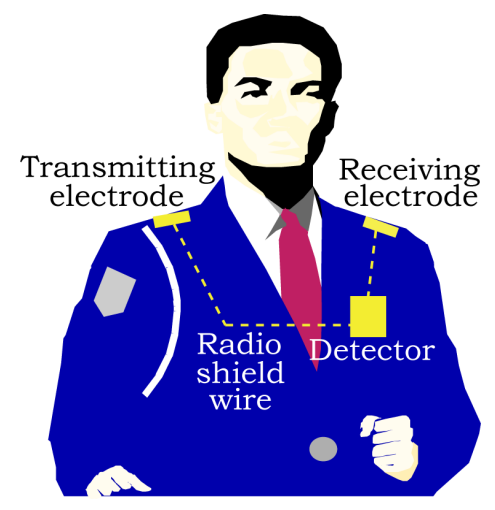

Figure 3: Installation of the dressing/undressing detection system.

Next, the mechanism of detecting dressing/undressing of the upper garment is explained. When the upper garment is put on, the electrodes and shoulders are closer and a signal loop consisting of detector $->$ shielded wire $->$ transmitting electrode $->$ human body $->$ receiving electrode $->$ shielded wire $->$ detector is formed. Thus, the detector will receive a signal transmitted by the detector itself. However, if the upper garment is taken off while a signal is transmitted and received, the electrodes pull away from the shoulders and the signal loop disappears, making the detector unable to receive the signal transmitted by it.

As explained above, it is possible to determine that the upper garment is put on when transmission/receiving of the signal is established and that the upper garment is not put on if otherwise. Thus, the detector and two electrodes can be used as a dressing/undressing detection system of the upper garment. In this section, the upper garment system was explained, but dressing/undressing detection of the lower garment is also possible if the detector and electrodes are installed in the lower garment.

\section{Implementation}

After the feasibility of the proposed system is confirmed with the basic experiment [10], the authors installed the system on an actual uniform. Figure 4 shows the system configuration, Figure 5 shows the prepared detector, and Figure 6 shows how the system installed in a dress shirt type uniform.

Specifications of the system are as follows.

1. Uniform used fits the shape of the tester for the experiments.

2. Power supply used is a 006P $9 \mathrm{~V}$ dry cell.

3. Electrodes are made of copper foil with its surface covered by a PVC sheet and a cloth patch and attached to the back of the uniform (at the shoulder) with hook-and-loop fasteners. 


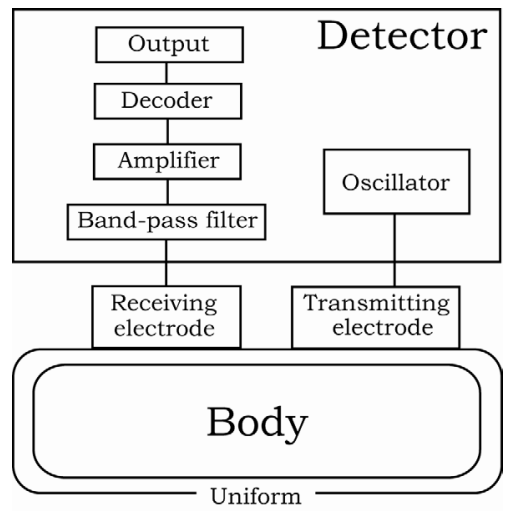

Figure 4: $\quad$ System configuration diagram.

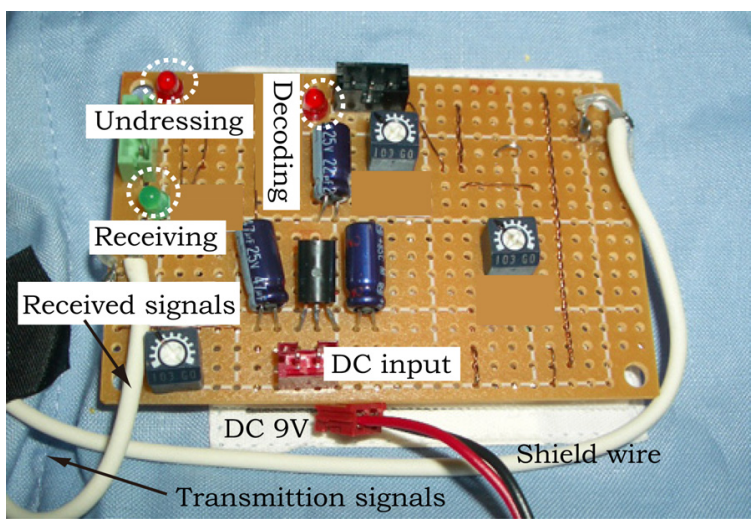

Figure 5: $\quad$ The prepared detector.

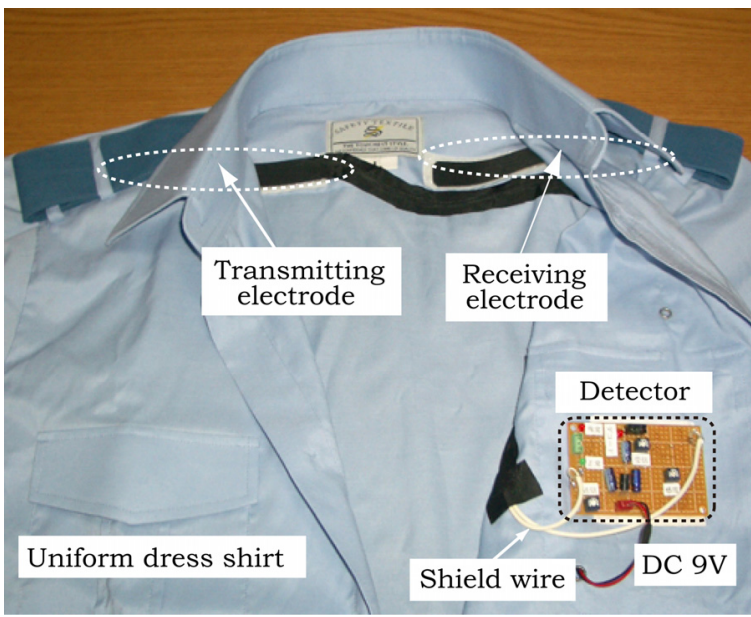

Figure 6: System implementation for uniform. 
4. The oscillator generates and sends $100 \mathrm{kHz}$ signals to the transmitting electrode.

5. Signals received by the receiving electrode are sent to the decoder through the bandpass filter and amplifier.

6. At the decoder, the transmitted signal and received signal are compared, and when both signals match, the decoder determines that intra-body communication is established (i.e. the uniform is put on) and illuminates LEDs labelled "Receiving" and "Decoding."

The decoder determines that the uniform is no longer on after interruption of intra-body communication for 5 seconds, and extinguishes the above two LEDs and illuminates the other LED labelled "Undressing." When intra-body communication is established again, the decoder will extinguish the "Undressing" LED and will illuminate the above two LEDs.

When dressing and undressing with the uniform was repeated by the tester wearing an undershirt for the experiment, the proposed system functioned exactly according to the above specifications. With this system installed in the jacket of the uniform, dressing and undressing with the jacket was repeated by the tester for the experiment, who was wearing an undershirt and a dress shirt, but the system functioned the same.

It was also tested while the uniform was put on to repeat (1) the up/down movement of both arms, (2) hopping of the body, and (3) jogging on the spot, and the system functioned the same. All these results show that the proposed system is highly practical and reliable.

\section{Considerations}

\subsection{Superiority of the proposed system}

This section provides an explanation that the problems associated with the dressing/undressing detection system using ultrasonic sensor [6] are eliminated in the proposed system.

First, the mechanism of the system using an ultrasonic sensor is explained. As shown in Figure 7, the Transmitter, Receiver A, and Receiver B are installed on the back of the upper garment so that Receiver A and Receiver B face together.

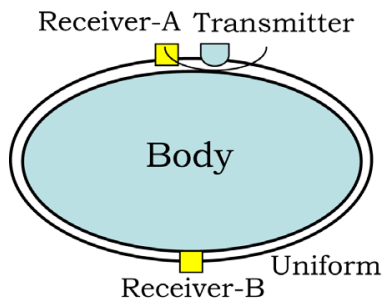

(A)

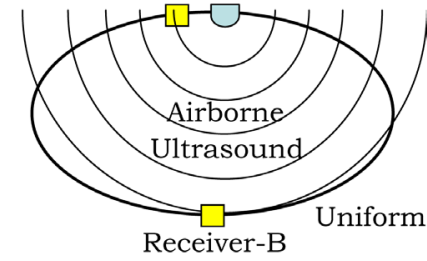

(B)

Figure 7: Dressing/undressing detection method using an ultrasonic sensor. 
The transmitter generates intermittent ultrasonic waves in short intervals in the semispherical direction.

As shown in Figure 7 (A), Receiver B will not receive ultrasonic waves from the transmitter when the jacket is on. This is because ultrasonic waves are reflected by the skin without penetrating into the body due to the difference in acoustic impedance between the air and the human body. Conversely, when the jacket is taken off as shown in Figure 7 (B), the Receiver B will receive ultrasonic waves from the Transmitter. Thus, this system determines whether the uniform is worn or not by identifying whether ultrasonic waves are received or not. However, the following problems are associated with this system.

1. Although the size of the transmitter and receivers are small, they are thick, and concentrated pressure on the body causes pain or discomfort to the person wearing the upper garment from external pressure on the upper garment.

2. If the Transmitter B fails and cannot receive ultrasonic waves while the uniform is put on, the system cannot identify whether the system failed (i.e., the system cannot detect that the uniform is taken off).

3. False undressing method: If a tubular object that is not transparent to ultrasonic waves is inserted between the body and the uniform when the upper garment is put on, and the upper garment and the tubular object are taken off at the same time, the system will not recognize the removal of the uniform because the Receiver B cannot receive ultrasonic waves.

In the proposed system, these problems are eliminated as follows.

1. The electrodes are thin and flexible and do not cause discomfort or pain from concentrated pressure on the human body when pressure is applied from outside the upper garment.

2. If either of the electrodes fails while the uniform is on, transmission/receiving of the signal is interrupted, and the system determines that the uniform has been removed. The system can notify the user of a failure with a buzzer or vibrator.

3. If the uniform and the tubular object are removed from the human body simultaneously, the signal loop disappears and transmission and receiving of the signal are interrupted. Thus, the system will recognize that the uniform has been removed.

As explained above, the proposed system eliminates the problems of the system using an ultrasonic sensor.

\subsection{Safety of the proposed system}

In the proposed system, high frequency signals are fed to the human body but no electrical shock is associated with those signals. Within the industrial and medical worlds, the following criteria are in place to avoid electrical shock.

Voltage: According to IEC 60950-1 (Information technology equipment Safety - Part 1: General requirements), it is specifically provided that peak voltage less than or equal to $42.4 \mathrm{~V}$ or DC voltage less than or equal to $60 \mathrm{~V}$ is called the safety extra-low voltage (SELV) and is not dangerous to the human body. 
Current: According to JIS T0601-1 (Medical electrical equipment - Part 1: General requirements for safety), it is specifically provided that electrical current less than or equal to $0.05 \mathrm{~mA}$ is not dangerous to the human body.

The reason we do not experience electrical shock when we use a body fat meter or a body composition meter is because these products employ voltage and current within the above criteria. Furthermore, the human body is susceptible to electrical shock with low frequency current but not susceptible to high frequency current.

1. Electric cautery uses a maximum of $1 \mathrm{~A}$ of electric current to the body, but the patient will not experience electric shock because the high frequency current from the hundreds of kilohertz to several megahertz range is injected to the body part [11].

2. The reason a person who uses a low frequency therapy device feels pain is because the device uses low frequency current less than $1 \mathrm{kHz}$ [12].

In the proposed system, current, voltage, and frequency in accordance with the above criteria are used, and the person wearing the uniform will not experience any electrical shock.

\subsection{Use of the conductive fabric}

In the actual installation, the surface of the copper foil is covered with PVC film and a patch of fabric. This is also necessary to prevent metal allergies (inflammation of the skin close to the electrode due to the chemical reaction between chlorine ions in sweat and copper). In practical application, conductive cloth is used for the electrodes so that a person wearing the uniform will not feel the electrodes installed in the uniform. Description of the method is as follows.

\subsubsection{Use of the silver fibre}

A patch of cloth woven from a string coated with silver [13] is used as an electrode. Silver has excellent conductivity and causes no harm to the human body; the material is used in tableware and artificial teeth.

\subsubsection{Method of installation}

As shown in Figure 8, the edge of the conductive fabric patch and one end of the shield wire are insulated, and the patch is sewn on the back of the upper garment (at the shoulders).

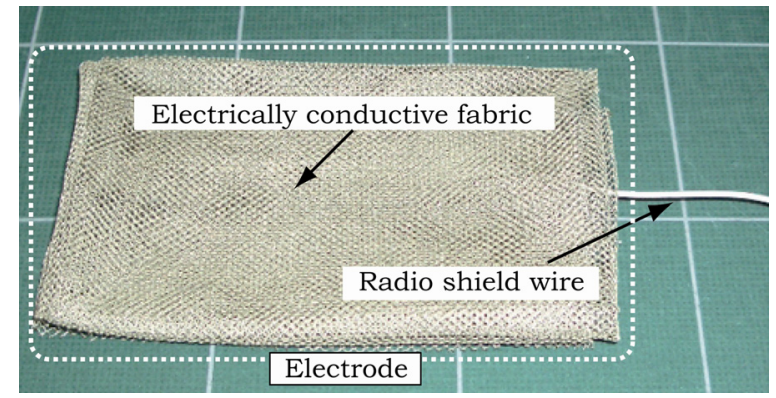

Figure 8: $\quad$ Electrode made of silver mesh fabric. 


\section{Conclusions}

Dressing/undressing detection system using intra-body communication technology that enables detection of dressing/undressing of the uniform was engineered and proposed. The system was installed in an actual uniform and practicality and high reliability were demonstrated, and the superiority and safety of the proposed system were explained. It is intended to further improve the system for practical application.

\section{Appendix}

The basic concept of intra-body communication is the same as the concept of wired communication. Generally, two conductive lines are required for wired communication. i.e., one is used as a signal line that transmits and receives the signal, and the other is used as a reference line to translate the reference potential. It is impossible to use the human body as two conductive lines because it is a single conductor, and only one conductive line is possible. So in the static coupling method, the human body is used as a signal line and static coupling is used as the reference line. Static coupling is the state where a capacitor equivalent is formed between the reference electrode and the space, and it provides the function as a conductive line for alternating signal, although it is not a visible conductor.

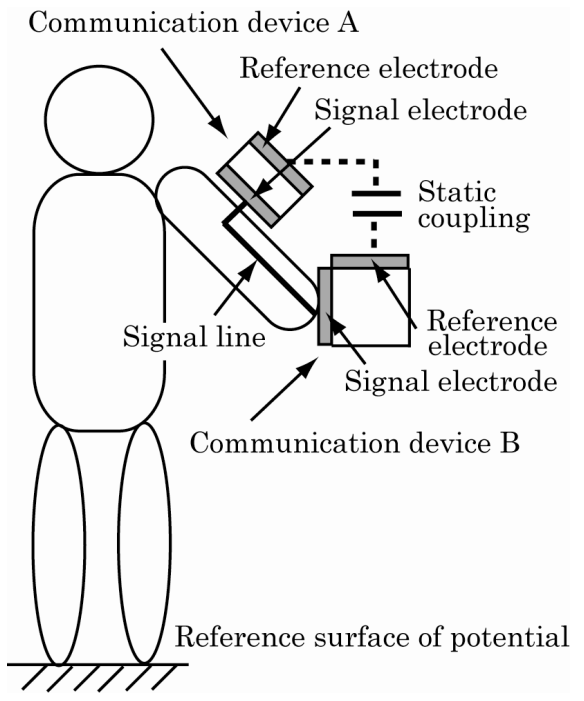

Figure 9: Image diagram of the intra-body communication technology by using the static coupling method.

\section{Acknowledgement}

A part of this study was supported by the Nippon Uniform Center. 


\section{References}

[1] Bell, R., Outrunning the law, http://www.trutv.com/library/ crime/criminal_mind/scams/frank_abagnale/

[2] The Washington Post, Alleged Fake Cop Had 'Police Station', http://www.washingtonpost.com/wp$\mathrm{dyn} /$ content/article/2007/02/10/AR2007021000288.html

[3] Mitnick, K., Simon, W. L., \& Wozniak, S., The Art of Deception: Controlling the Human Element of Security". John Wiley \& Sons, 2002.

[4] Global Security.org, Identification Friend or For (IFF) System, http://www.globalsecurity.org/military/systems/aircraft/systems/iff.htm

[5] NEWS.SCOTSMAN.COM: Officer sacked for selling helmet, Published Date: 17 May 2008, http://news.scotsman.com/aberdeen/Officer-sackedfor-selling-helmet.4094052.jp

[6] Fujikawa, M., Aoki, H., Nishigaki, M., Yoshizawa, M. \& Tsujii, S., Design of Office Security System that is capable of Detecting Unauthorized Persons Wearing Office Uniforms, Proc. of the 2nd Int'l Conf. on Safety and Security Systems in Europe, 2007.

[7] Doi, K. \& Nishimura, T., Technologies for Personal Fitness: HighReliability Communication Technology Using Human Body as Transmission Medium, MEW Technical Report (Japanese Text), http://sciencelinks.jp/j-east/article/200519/000020051905A0765593.php

[8] Nippon Telegraph and Telephone Corporation (NTT), What's Red Tacton? http://www.redtacton.com/en/info/index.html

[9] Kato, Y., Akioka, S. \& Mitsubayashi, K., Heart rate Measurement by Ubiquitous Human body Communication, http://sciencelinks.jp/jeast/article/200603/000020060305A0916340.php (Japanese Text)

[10] Fujikawa, M., Nishigaki, M., Yoshizawa, M., Furusawa, K. \& Tsujii, S., Proposal of uniform dressing/undressing detection system using bodybased data transmission technology - To prevent a crime abusing office uniforms -. Proc. of the Domestic Symposium on Computer Security, pp. 701-706, 2008.

[11] Medical \& biological system, Lecture Note (Vol.11), Biological properties and Safety, www.t-net.ne.jp/ kondoy/lecture/bme/11.pdf (Japanese Text)

[12] NIHON MEDIX, Low-frequency massage device (Japanese Text), http://www.nihonmedix.co.jp/page/reha/reha_page01-02.html

[13] Less EMF Inc.: High performance silver mesh fabric, http://www.lessemf. com/fabric.html 